\title{
Electrochemical Impedance Analysis of Biofunctionalized Conducting Polymer-Modified Graphene-CNTs Nanocomposite for Protein Detection
}

\author{
Shobhita Singal ${ }^{1,2} \cdot$ Avanish K. Srivastava $^{1} \cdot$ Rajesh $^{1,2}$
}

Received: 28 June 2016/ Accepted: 23 August 2016/Published online: 15 September 2016

(C) The Author(s) 2016. This article is published with open access at Springerlink.com

\begin{abstract}
We report an electrodeposited poly(pyrrole-co-pyrrolepropylic acid) copolymer modified electroactive graphene-carbon nanotubes composite deposited on a glassy carbon electrode to detect the protein antigen (cTnI). The copolymer provides pendant carboxyl groups for the site-specific covalent immobilization of protein antibody, antitroponin I. The hybrid nanocomposite was used as a transducer for biointerfacial impedance sensing for cTnI detection. The results show that the hybrid exhibits a pseudo capacitive behaviour with a maximum phase angle of $49^{\circ}$ near $1 \mathrm{~Hz}$, which is due to the inhomogeneous and porous structure of the hybrid composition. The constant phase element of copolymer is $0.61(n=0.61)$, whereas, it is $0.88(n=0.88)$ for the hybrid composites, indicating a comparatively homogeneous microstructure after biomolecular functionalization. The transducer shows a linear change in charge transfer characteristic $\left(R_{\mathrm{et}}\right)$ on cTnI immunoreaction for spiked human serum in the concentration range of $1.0 \mathrm{pg} \mathrm{mL}^{-1}$ $10.0 \mathrm{ng} \mathrm{mL}^{-1}$. The sensitivity of the transducer is $167.8 \pm 14.2 \Omega \mathrm{cm}^{2}$ per decade, and it also exhibits high specificity and good reproducibility.
\end{abstract}

Keywords Conducting polymer - Graphene - Carbon nanotube · Hybrid · Transducer · Protein antigen cTnI · Electrochemical impedance

\section{Introduction}

In recent years, enormous interest has been attached to hybrid nanocomposites due to their wide applications in environmental science, energy conversion, sensing, etc. [1]. Recently, theoretical and experimental reports on hybrid carbon nanostructures have brought special interest

Electronic supplementary material The online version of this article (doi:10.1007/s40820-016-0108-2) contains supplementary material, which is available to authorized users.

\section{Rajesh}

rajesh_csir@yahoo.com

1 CSIR-National Physical Laboratory, Dr. K. S. Krishnan Road, New Delhi 110012, India

2 Academy of Scientific and Innovative Research (AcSIR), CSIR-National Physical Laboratory, Dr. K. S. Krishnan Road, New Delhi 110012, India in design and fabrication of conducting polymer/carbon nanostructured composites $[2,3]$. The interest is motivated by their extraordinary physical properties and remarkable electrocatalytic properties. Polypyrrole (PPy), a conducting polymer with high conductivity and excellent biocompatibility, has been widely used as a functional material in the development of electrochemical sensors [4-8]. However, it has poor permeability and hydrophobicity that obstructs the diffusion of analyte, leading to a fall off sensitivity of the immunosensor. Another major drawback is associated with its instability due to swelling and exfoliation with times upon electrochemical modulation and/or solution soaking [9]. However, the deposition of PPy film on highly conductive and rigid material like graphene or metal nanoparticle is proved to be an efficient way to enhance the electrochemical/mechanical stability of the polymer film without compromising its electrochemical activity $[10,11]$.

Carbon nanomaterials, including carbon nanotubes (CNTs) and graphene, have shown great potential in 
biosensing due to its excellent electric/thermo conductivity, good mechanical/chemical stability, high surface area, and unique physical/chemical properties [12-17]. In graphene, these excellent properties only emerge in the 2D planar direction, thereby limiting its scope and applications. New efforts in graphene researches have attempted to address this weakness, wherein graphene acts as a platform for anchoring other nanomaterials. CNTs, whose properties emerge in the axial direction, can be functionalized onto the surface of graphene, which can combine unique properties of two carbon allotropes in all directions and increase active surface areas and faster electron transfer. Recent studies predict that three dimensional graphene-CNTs (G-CNTs) nanostructure possesses desirable out-of-plane properties when maintaining in-plane properties. It is attractive for numerous innovative applications, including as an efficient transducer for sensor applications [18-22].

Cardiac troponin I (cTnI), a protein subunit of cardiac troponin complex is extensively used as diagnostic marker of acute myocardial infarction (AMI) [23]. Owing to its small size $(29 \mathrm{kDa})$, cTnI rises rapidly in the blood stream within 3-4 h after onset of AMI and remains to be raised up to 10-14 days. This will result in a long diagnostic window for the detection of AMI. Compared with other biomarkers of myocardial injury, such as myoglobin, C-reactive protein and creatine kinase-MB isoenzyme, cTnI has excellent cardio specificity and selectivity for cardiac damage other than damage to skeletal muscle or other organs. A cTnI level of $0.1-2.0 \mathrm{ng} \mathrm{mL}^{-1}$ indicates to an unstable angina and other heart disorder, whereas a level greater than $2.0 \mathrm{ng} \mathrm{mL}^{-1}$ places a patient in high-risk category for early adverse outcomes [24, 25]. This underlines the need to develop a sensitive diagnostic tool for quantitative detection of $\mathrm{cTnI}$ for diagnosis of AMI.

In the present work, we demonstrate the electrochemical synthesis of conducting copolymer PPy-PPa over G-MWCNT hybrid film deposited on a glassy carbon electrode (GCE), as an impedance immunosensor, for ultrasensitive detection of cardiac troponin I (cTnI) spiked in human serum. The surface morphology and electrochemical properties of the hybrid were characterized. The analytical performance of the bioelectrode was investigated for the quantitative detection of cTnI in spiked human serum using charge transfer characteristic $\left(R_{\mathrm{et}}\right)$ as a sensing element in electrochemical impedance spectroscopy. The utilization of the changes observed in the $R_{\mathrm{et}}$ provided a good correlation with antigen concentration in the lowfrequency region $(<1 \mathrm{~Hz})$, which helps in preserving the micro environment for biomolecular reactions useful for both in-field and at-line applications.

\section{Experimental}

\subsection{Chemicals and Reagents}

Human cardiac troponin I (cTnI: Cat 8T53), human anticardiac troponin I (anti-cTnI: Cat 4T21 MAb19C7), C-reactive protein (CRP: Cat $8 \mathrm{C} 72)$, and myoglobin (cMb: Cat 8M50) were obtained from Hytest (Turku, Finland). $\mathrm{N}$-(3dimethylaminopropyl)- $N^{\prime}$-ethyl carbodiimide hydrochloride (EDC), $N$-hydroxysuccinimide $98 \%$ (NHS), pyrrole, pyrrolepropylic acid, and $p$-toulenesulfonic acid ( $p$ TSA) were obtained from Sigma-Aldrich Corp. All other chemicals were of analytical grade and used without further purification.

\subsection{Instruments}

The surface morphologies and electrochemical characterizations of the bioelectrode were carried out using scanning electron microscopy (SEM, SUPRA40 VP, Germany), highresolution transmission electron microscopy (HRTEM, Technai G2F30 STwin, The Netherlands), Raman spectroscopy (Renishaw Raman spectrometer, Germany), and energy dispersive X-ray analysis (EDAX, SUPRA40 VP, Germany). Electrochemical impedance spectroscopic (EIS) measurements were done on an AUTOLAB instrument from Eco Chemie (PGSTAT302N, The Netherlands). The EIS measurements were conducted in $0.1 \mathrm{M}$ phosphate buffer solution (PBS) (pH 7.4, 0.1 M KCl) containing a mixture of $2.0 \mathrm{mM} \mathrm{K}_{3}\left[\mathrm{Fe}(\mathrm{CN})_{6}\right]$ and $2.0 \mathrm{mM} \mathrm{K}_{4}\left[\mathrm{Fe}(\mathrm{CN})_{6}\right]$. The EIS parameters were obtained by circuit fitting the EIS experimental data using GPES (General purpose electrochemical system version 4.9, Eco Chemie) software. All electrochemical measurements were carried out in a conventional three-electrode cell configuration consisting of a PPy-PPa/ G-CNTs/GCE as a working electrode, $\mathrm{Ag} / \mathrm{AgCl}$ as a reference electrode, and platinum wire as a counter electrode.

\subsection{Preparation of the Bioelectrode}

The G-CNTs hybrid films were synthesized by the chemical vapour deposition (CVD) technique as reported earlier [26]. In brief, the G-CNTs hybrid was grown on a copper foil deposited $\sim 1 \mathrm{~nm}$ thickness of Fe film using e-beam evaporator. The Fe-deposited copper foil was placed in the fused silica tube $(5 \mathrm{~cm}$ in diameter and $100 \mathrm{~cm}$ in length) and the temperature was raised to $750{ }^{\circ} \mathrm{C}$ under $200 / 100$ sccm $\mathrm{Ar} / \mathrm{H}_{2}$ atmosphere flow. A $10 \mathrm{sccm}$ flow of acetylene (used as a gaseous carbon source) was introduced for 20 min once the temperature stabilized at $750{ }^{\circ} \mathrm{C}$, followed by cooling of the furnace to the room temperature in $\mathrm{Ar} / \mathrm{H}_{2}$ atmosphere flow. The growth time of $20 \mathrm{~min}$ is the most 
optimized at which the CNTs are uniform all over the graphene surface. If the time is less than $20 \mathrm{~min}$ (e.g., 10 or $15 \mathrm{~min}$ ), the CNTs are un-uniformed. Whereas, if the time is more than $20 \mathrm{~min}$, it will get a high density CNTs, which could not hold firmly onto the GCE surface and peel off during experimental measurements.

For the preparation of PPy-PPa/G-CNTs nanocomposite, GCE (3 $\mathrm{mm}$ in diameter) was first polished with respective $1,0.3$, and $0.05 \mu \mathrm{m}$ alumina slurry using a polishing cloth to produce a mirror-like surface, and then it was rinsed ultrasonically with double distilled water and ethanol for 3 min each, and dried with a high purity $\mathrm{N}_{2}$ gas flow. G-CNTs hybrid film was transferred over GCE by scooping, followed by drying in oven at $50{ }^{\circ} \mathrm{C}$ for $1 \mathrm{~h}$. The G-CNTs-modified GCE were anodized at $1.7 \mathrm{~V}$ for $500 \mathrm{~s}$ in PBS ( $\mathrm{pH} 7.4,0.1 \mathrm{M} \mathrm{KCl})$, followed by cathodization at $-0.6 \mathrm{~V}$ for $60 \mathrm{~s}$ to obtain an electroactive G-CNTs. The PPy-PPa copolymer was electrochemically deposited on the G-CNTs/GCEs in a degassed $\left(\mathrm{N}_{2}\right.$ purged) aqueous solution containing $0.1 \mathrm{M}$ pyrrole, $0.03 \mathrm{M}$ pyrrolepropylic acid, and $0.1 \mathrm{M} p$ TSA at a fixed current density of $1 \mathrm{~mA} \mathrm{~cm}{ }^{-2}$ by inducing a total charge density of $100 \mathrm{mC} \mathrm{cm}^{-2}$. The most optimum-induced charge density of $100 \mathrm{mC} \mathrm{cm}^{-2}$ was chosen based on the facts that at low density $\left(<100 \mathrm{mC} \mathrm{cm}^{-2}\right)$, the polymer film is unstable, whereas, at large density $\left(>100 \mathrm{mC} \mathrm{cm}^{-2}\right)$, the electroactive polymer surface is too small. Finally, the PPy-PPa/GCNTs/GCE was biofunctionalized with anti-cTnI by incubating with an aqueous solution containing $0.03 \mathrm{M}$ NHS and $0.15 \mathrm{M}$ EDC for $1 \mathrm{~h}$ and then with $10 \mu \mathrm{L}$ PBS containing $100 \mu \mathrm{g} \mathrm{mL}^{-1}$ anti-cTnI at $4{ }^{\circ} \mathrm{C}$ for $2 \mathrm{~h}$, followed by washing with PBS and drying under $\mathrm{N}_{2}$ gas flow. The biofunctionalized electrode was then incubated in $0.1 \%$ bovine serum albumin (BSA) solution (w/v) to block the non-specific binding sites, if any, on the electrode surface, followed by washing with PBS, to remove the physically adsorbed antibody and drying under $\mathrm{N}_{2}$ gas flow to obtain the desired anti-cTnI-PPy-PPa/G-CNTs/GCE bioelectrode. The as-prepared bioelectrode was stored at $4{ }^{\circ} \mathrm{C}$ when not in use. The stepwise fabrication of the bioelectrode is schematically represented in Fig. 1.

\section{Results and Discussion}

\subsection{Microstructural Characterization of PPy-PPa/ G-CNTs Nanocomposite}

Figure 2a shows the SEM image of G-CNTs after the PPy$\mathrm{PPa}$ copolymer electrodeposition (the inset is G-CNTs image). It can be seen in the inset that CNTs are highly dense and uniformly distributed on the graphene surface. After copolymer electrodeposition, three-dimensional petal-like structure was formed uniformly across the G-CNTs hybrid surface. This was further confirmed by EDAX spectra, showing the characteristic peaks (Fig. 2b), and elemental mapping (Fig. 2c-f) corresponding to energy levels of carbon $(0.277 \mathrm{keV})$, nitrogen $(0.392 \mathrm{keV})$, oxygen $(0.525 \mathrm{keV})$, and sulphur $(2.307 \mathrm{keV})$. The presence of $\mathrm{N}, \mathrm{O}$, and $\mathrm{S}$ in the elemental analysis due to amine, carboxyl, and sulphonate groups in PPy-PPa copolymer, revealed the uniform electrodeposition of copolymer throughout the surface of G-CNTs.

HRTEM images of PPy-PPa/G-CNTs composite are displayed in Figs. 2g-i. The polymer, graphene, and CNTs were observed (Fig. 2g) clearly. In the encircled region of Fig. 2a, the gray-level contrast may be corresponding to graphene, whereas, the petal shape is from PPy-PPa which has a clear distinct gray-level difference from CNTs and graphene due to its thick polymer-chain composition. Selected area electron diffraction (SAED, inset 1 in Fig. $2 \mathrm{~g}$ ) pattern exhibits a set of diffused Debye rings taken from the encircled composite. Due to the complexity of the hybrid (graphene, CNTs and PPy-PPa) with several interfaces and grain boundaries, it is difficult to interpret the pattern further. However, it still can infer that the composite has a major fraction of crystallinity along with a minor amorphous structure. At high magnifications, the presence of graphene is clear and multilayered graphene is confirmed. In Fig. 2h, three layers of graphene (marked as I, II, III) are overlapped. At the atomic scale, a typical honeycomb structure with the fringe separation of about $0.32 \mathrm{~nm}$ is clearly observed (inset 2 in Fig. $2 \mathrm{~h}$ ). The SAED from graphene exhibits a hexagonal arrangement of diffraction spots in reciprocal space (inset 3 in Fig. 2h).

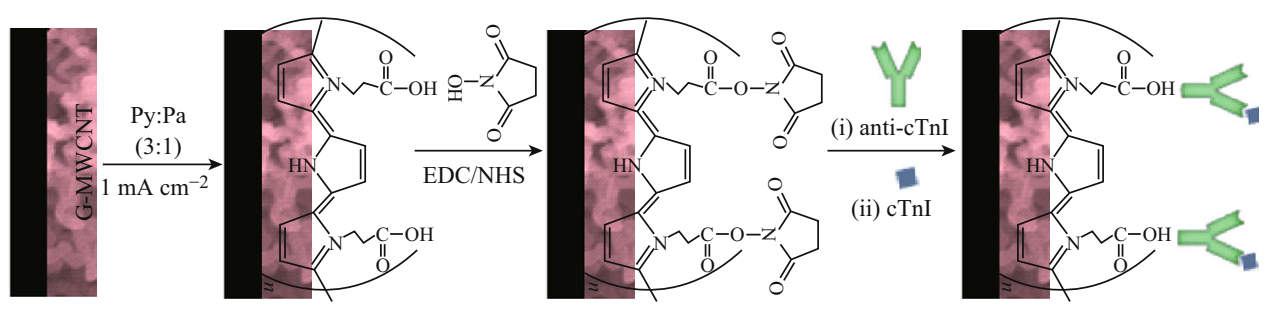

Fig. 1 Fabrication scheme of anti-cTnI-PPy-PPa/G-CNTs/GCE bioelectrode 

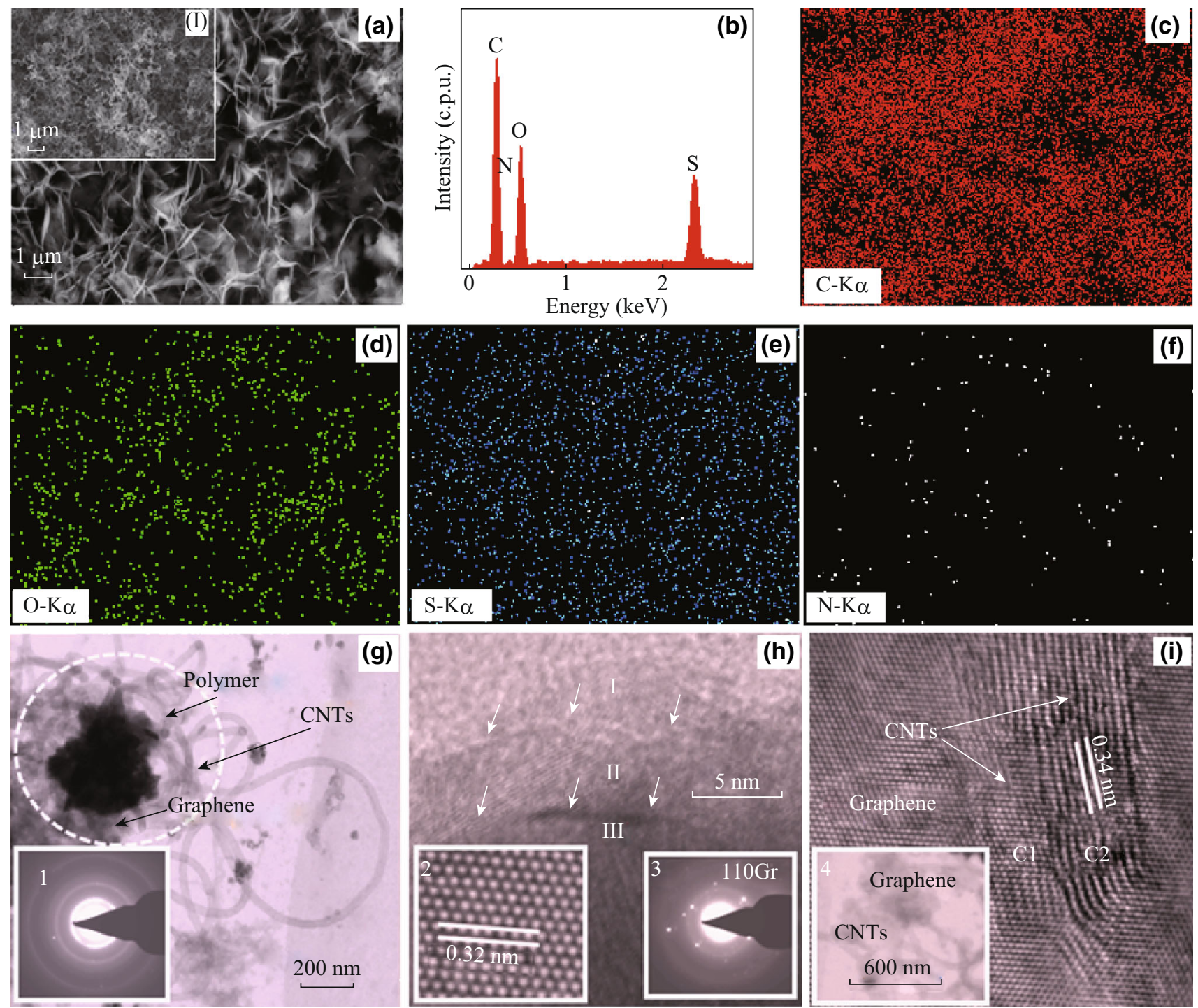

Fig. 2 a SEM image, b EDAX spectrum, c-f elemental map, g-i HRTEM images of PPy-PPa/G-CNTs composite. Insets (I) SEM image of G-CNTs, (1) SAED pattern of the composite, (2) Atomic scale image of graphene, and (3) SAED pattern of graphene, (4) TEM image of interface between graphene and CNTs at initial growth stage

Miller indices of (110) plane corresponding to a hexagonal crystal structure is marked on the SAED pattern. Figure $2 \mathrm{i}$ displays CNTs structure overlapped in two nanotubes, marked as $\mathrm{C} 1, \mathrm{C} 2$ on nano-sheets of graphene. The individual walls of the nanotubes of interlayer separation about $0.34 \mathrm{~nm}$ are stacked with a very coherent microstructure of graphene throughout the region. The TEM image of the interface between graphene and CNTs (inset 4 in Fig. 2i) shows the growing feature of the CNTs on the graphene floor during initial CVD growth process. Raman spectroscopy shown in Fig. S1 exhibits the characteristic 2D and $\mathrm{G}$ peaks with an intensity ratio $\left(I_{2 \mathrm{D}} / I_{\mathrm{G}}\right)$ of 0.35 , and further revealed a trilayer graphene thickness in G-CNTs hybrid.

\subsection{Electrochemical Characterization of PPy-PPa/ G-CNTs and the Bioelectrode}

Linear sweep voltammetry (LSV) is highly efficient and sensitive technique to investigate the electron transfer properties of the electrode surface, and it was utilized to characterize the anti-cTnI-PPy-PPa/G-CNTs/GCE electrode here. The linear sweep voltammetric measurements were performed over the voltage range of $0-0.5 \mathrm{~V}$ versus $\mathrm{Ag} / \mathrm{AgCl}$ as reference electrode in PBS containing $2.0 \mathrm{mM}$ $\left[\mathrm{Fe}(\mathrm{CN})_{6}\right]^{3-/ 4-}$ at a scan rate of $50 \mathrm{mV} \mathrm{s}^{-1}$. Figure $3 \mathrm{a}$ shows anodic peak in LSV conducted on the electrode at various stages of surface modifications. The current density $(J)$ is $0.38 \mathrm{~mA} \mathrm{~cm}^{-2}$ for bare GCE and it increases to 

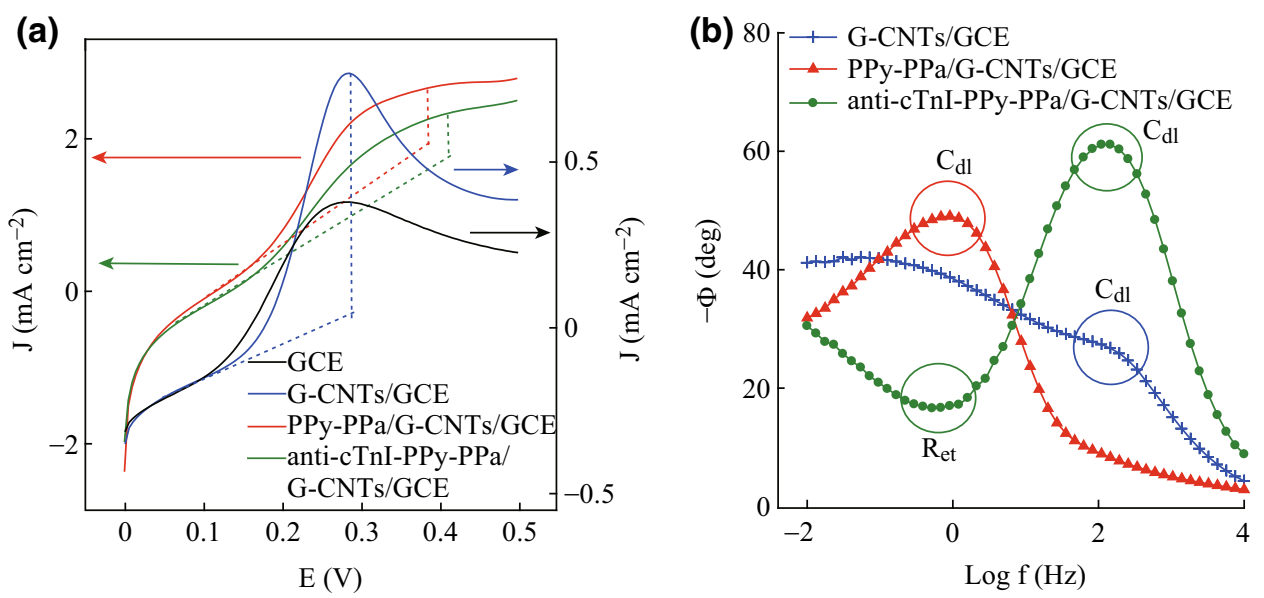

Fig. 3 a Linear sweep voltammogram of the hybrid bioelectrode at different stages of modification surface. b Corresponding Bode plot

$0.72 \mathrm{~mA} \mathrm{~cm}^{-2}$ for the electroactive G-CNTs/GCE. This is due the increased electron transfer characteristics resulted from the edge plane defects at G-CNTs, which generated from the oxygenated species during electrode anodization. After electrodeposition of PPy-PPa copolymer on G-CNTs/ GCE electrode, the value of $J$ decreases to $0.65 \mathrm{~mA} \mathrm{~cm}^{-2}$ with a large background current [27]. This may be attributed to the presence of negatively charged $-\mathrm{COOH}$ group of $\mathrm{PPa}$, which generates a repulsive force towards the negatively charged redox probe at the electrode/solution interface, and thus reduces the ion perturbation. The electroactive surface area of G-CNTs hybrid film was found to be $16.1 \times 10^{-5} \mathrm{~cm}^{2}$ calculation using Randles-Sevcik equation, which slightly decreases to $14.5 \times 10^{-5} \mathrm{~cm}^{2}$ after modification with the PPy-PPa. The detail is described in supporting information. After biomolecular immobilization with anti-cTnI and subsequent passivation of the electrode with a blocking reagent BSA, further decreases in $J$ values were observed to be 0.54 and $0.42 \mathrm{~mA} \mathrm{~cm}^{-2}$, respectively. This may be explained on the basis of the insulating and highly organized hydrophobic layer of protein molecules at the bioelectrode surface, which could hinder the access of the redox probe on electrode surface and thus result in sluggish electron transfer kinetics.

The above configuration was further characterized by EIS. Briefly, in EIS theory, a Nyquist or Bode plot is mainly composed of three regions: the high-frequency part corresponding to the solution properties, the middle-frequency part corresponding to the electrode/solution interface (i.e., capacitive properties), and the low-frequency part corresponding to the charge transfer characteristics of the electrode surface. The complex impedance can be represented by Eq. 1,

$Z^{\prime}=Z e^{i \Phi}$,

where the magnitude $Z$ represents the ratio of the voltage difference amplitude to the current amplitude and acts like resistance, and $\Phi$ gives the phase difference between the voltage and the current. The change in the phase shift (angle $\Phi)$ in the Bode plot was chosen as a prominent EIS element in frequency region of $0.01-10,000 \mathrm{~Hz}$ as shown in Fig. $3 \mathrm{~b}$. The G-CNTs electrode shows two phase shifts of $\Phi=26.6^{\circ}$ and $39.2^{\circ}$ at frequency of $\sim 162$ and $\sim 1 \mathrm{~Hz}$, respectively. These phase shifts at higher and lower frequency correspond to capacitive and diffusive characteristics of the G-CNTs hybrid. However, a single-phase shift $\left(\Phi=49^{\circ}\right)$ at frequency of $\sim 1 \mathrm{~Hz}$ was observed for the PPy-PPa copolymer modified G-CNTs, which corresponds to only a capacitive behaviour with no diffusive characteristic, indicating a moderate heterogeneous feature of the modified electrode compared with its native G-CNTs. This was further enunciated by another impedance parameter of constant phase element $n$, which accounts for the surface inhomogeneity of the electrode surface. The value of $n=1$ represents a smooth defect free surface, whereas $0<n<1$ represents porous and rough surface [28]. The high value of $n=0.61$ observed on PPy-PPa/G-CNTs compared with $\mathrm{n}=0.44$ on native G-CNTs further indicates a slight reduction in surface inhomogeneity in the polymer-modified electrode, which is later found to be nearly homogenous $(n=0.88)$ upon biomolecular immobilization with anti-cTnI. The same is reflected in the change of phase angle, where the maximum phase shift $\left(\Phi=61^{\circ}\right)$ obtained in G-CNTs at frequency of $\sim 1.0 \mathrm{~Hz}$ is moved to a high region $(>100 \mathrm{~Hz})$. A lowest phase shift (where $v / t$ and $I / t$ are nearly in phase) with $\Phi=29^{\circ}$ appears at the same frequency, corresponding to a charge transfer characteristic of a homogeneous surface after biomolecular immobilization.

\subsection{EIS Response of the Bioelectrode to Protein Antigen cTnI}

When biomolecular probe (antibody) forms a complex with the complementary antigen molecule upon immunoreaction 

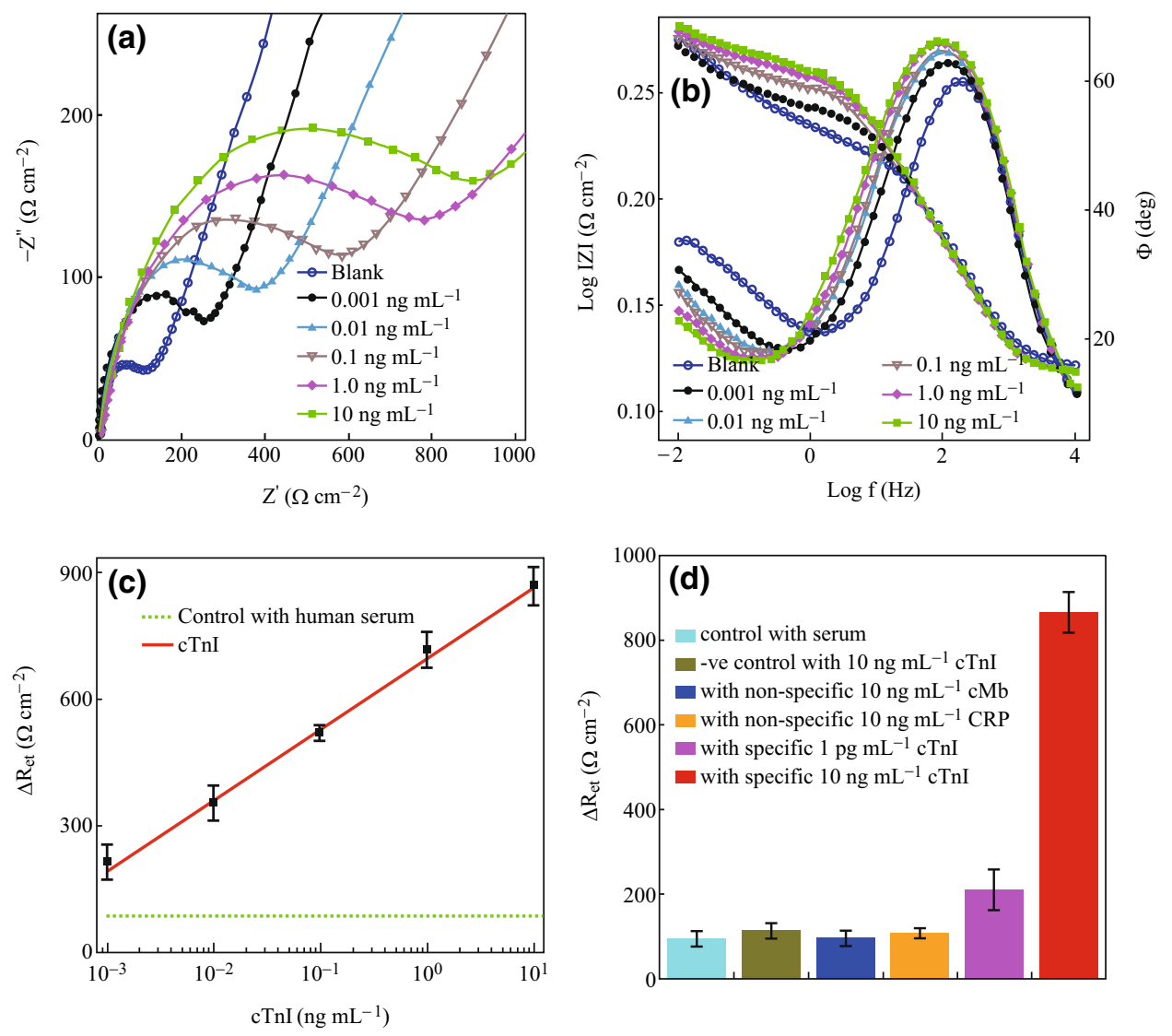

Fig. 4 a Nyquist plot of the bioelectrode before and after incubation with different cTnI concentrations in human serum. b Corresponding Bode plot. c Concentration-dependent calibration curve of the bioelectrode. d Specificity of the bioelectrode towards cTnI with multiple controls

on the bioelectrode surface, it will cause an increase in charge transfer resistance that leads to a decrease in the phase shift (lowest phase angle) of the circuit in low-frequency region [29]. The anti-cTnI-PPy-PPa/G-CNTs/GCE bioelectrode was incubated with a $10 \mu \mathrm{L}$ sample of cTnI-spiked human serum for $10 \mathrm{~min}$ at room temperature, followed by washing with PBS and drying under $\mathrm{N}_{2}$ gas flow. Impedance analysis was carried out in PBS solution containing $2.0 \mathrm{mM}$ $\left[\mathrm{Fe}(\mathrm{CN})_{6}\right]^{3-14-}$.

Figure 4 shows the Nyquist and the Bode plot of the bioelectrode with different cTnI concentrations. The diameter of the semicircle in the Nyquist plot of the bioelectrode (Fig. 4a) is gradually increased with increasing concentration of the dispensed cTnI, indicating an increasing antigen-antibody complex formation. This will result in a large electron transfer resistance $\left(R_{\mathrm{et}}\right)$ at the electrode surface. Since the changes in $R_{\mathrm{et}}$ values were found to be much more prominent than those of other impedance components (Table 1), it was taken as a suitable sensing parameter. Though, not many changes were observed in the $Z_{\mathrm{w}}$ values, noticeable changes in $Y_{\mathrm{o}}$ in a decreasing order show decreasing capacitive behaviour on subsequent immunoreaction with increasing concentration of cTnI. This may be due to the increasing coverage of electrode surface with increasing cTnI concentration and decreasing its exposure to electrolyte [30]. The immunoreaction was further investigated by corresponding Bode plot (Fig. 4b). It is interesting to note that the lowest phase angles on immunoreaction were observed at lowfrequency region of $0.1-1.0 \mathrm{~Hz}$, which corresponds to an increasing $R_{\text {et }}$ feature of the bioelectrode, indicating to a good probe (anti-cTnI) orientation and biocompatible feature of the electrode.

Figure $4 \mathrm{c}$ shows the calibration curve of the bioelectrode. One can see a linear relationship between the changes in charge transfer resistance i.e., before (blank) and after immunoreactions $\left[\Delta R_{e t}=\left(R_{e t}\right)_{\text {after immunoreaction }}-\left(R_{e t}\right)_{\text {blank }}\right]$, and the cTnI concentration is in the range of $0.001-$

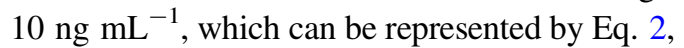

$\Delta R_{\mathrm{et}}[\mathrm{cTnI}]=b[\mathrm{cTnI}]+695.8 \pm 18.96$.

The bioelectrode shows $R_{\text {et }}$ sensitivity (slope 'b' of the calibration curve) of $167.8 \pm 14.2 \Omega \mathrm{cm}^{2}$ per decade of cTnI. The analytical performance of the bioelectrode is compared with the previous reports (Table 2) and was found to be better in terms of linear dynamic range and 
Table 1 EIS characteristic parameters of the bioelectrode on immunoreaction with different concentrations of target cTnI

\begin{tabular}{|c|c|c|c|c|c|c|}
\hline $\begin{array}{l}\text { cTnI concentration } \\
\left(\mathrm{ng} \mathrm{mL} \mathrm{m}^{-1}\right)\end{array}$ & $R_{\mathrm{et}}\left(\Omega \mathrm{cm}^{2}\right)$ & $\mathrm{CPE} \mathrm{Y}_{\mathrm{o}}\left(\mathrm{mF} \mathrm{cm}^{-2}\right)$ & $n$ & $Z_{\mathrm{w}}\left(\times 10^{-5}\right)\left(\Omega \mathrm{cm}^{2}\right)$ & $\chi^{2}\left(\times 10^{-2}\right)$ & $\begin{array}{l}\text { RSD (\%) in } \\
\Delta R_{\text {et }}(n=3)\end{array}$ \\
\hline Blank & 109.2 & 1.98 & 0.824 & 5.25 & 3.62 & - \\
\hline 0.001 & 298.6 & 1.61 & 0.843 & 4.78 & 3.59 & 13.4 \\
\hline 0.010 & 416.5 & 1.42 & 0.844 & 4.70 & 5.37 & 11.3 \\
\hline 0.10 & 616.0 & 1.35 & 0.793 & 4.76 & 6.53 & 2.8 \\
\hline 1.00 & 793.1 & 1.26 & 0.791 & 4.73 & 5.36 & 6.0 \\
\hline 10.0 & 925.4 & 1.13 & 0.797 & 4.73 & 4.84 & 5.3 \\
\hline
\end{tabular}

Table 2 Comparison of analytical performance of the bioelectrode with other existing biosensor

\begin{tabular}{|c|c|c|c|c|}
\hline Sensing technique & Transducing matrix & Detection range & Detection limit & References \\
\hline Amperometry & $\mathrm{PDMS} / \mathrm{Au}$ & $0.2 \mathrm{ng} \mathrm{mL}^{-1}-10.0 \mu \mathrm{g} \mathrm{mL}^{-1}$ & $148.0 \mathrm{pg} \mathrm{mL}^{-1}$ & [2] \\
\hline Impedimetric & Carbon nanofiber & $0.25-1.0 \mathrm{ng} \mathrm{mL}^{-1}$ & $0.2 \mathrm{ng} \mathrm{mL}^{-1}$ & {$[31]$} \\
\hline Surface plasmon resonance & $\mathrm{Au}$ nanorods & - & $10.0 \mathrm{pg} \mathrm{mL}^{-1}$ & {$[32]$} \\
\hline Potentiometry & $\mathrm{Au} / \mathrm{ITO}$ & $1.0-100.0 \mathrm{ng} \mathrm{mL}^{-1}$ & - & {$[33]$} \\
\hline Stripping voltammetry & $\mathrm{Ag} / \mathrm{SPE}$ & $0.1-32.0 \mathrm{ng} \mathrm{mL}^{-1}$ & $0.10 \mathrm{ng} \mathrm{mL}^{-1}$ & [34] \\
\hline Colorimetric & $\mathrm{PDMS} / \mathrm{Au}$ & $0.01-10.0 \mathrm{ng} \mathrm{mL}^{-1}$ & $0.01 \mathrm{ng} \mathrm{mL}^{-1}$ & [35] \\
\hline Lateral flow assay & Magnetic beads & - & $0.01 \mathrm{ng} \mathrm{mL}^{-1}$ & {$[36]$} \\
\hline Impedimetric & PPy-Ppa/G-CNTs & $1.0 \mathrm{pg} \mathrm{mL}^{-1}-10.0 \mathrm{ng} \mathrm{mL}^{-1}$ & $1.0 \mathrm{pg} \mathrm{mL}^{-1}$ & $\begin{array}{l}\text { Present } \\
\text { work }\end{array}$ \\
\hline
\end{tabular}

lowest detection limit $[2,31-36]$. This better performance of the bioelectrode may be attributed to the high cTnI probe loading due to active pendant carboxyl binding sites of the conducting PPy-PPa and electroactive behaviour of the G-CNTs hybrid, resulting in enhanced electron transfer at the electrode solution interface of the bioelectrode on immunoreaction.

The specificity of the bioelectrode towards specific $\mathrm{cTnI}$ was examined by monitoring the responses from multiple controls such as native normal human serum, non-specific biomarkers C-reactive protein (CRP), myoglobin (cMb) spiked in human serum, and a negative control with a native electrode (PPy-PPa/G-CNTs/GCE) without anticTnI immobilization. The comparative response of the bioelectrode with multiple controls with respect to the specific cTnI under identical condition is shown in Fig. 4d. The response of $\Delta R_{\mathrm{et}}=98.6 \pm 13.0$ and $111.3 \pm 7.3 \Omega$ $\mathrm{cm}^{2}$ obtained for $10 \mathrm{ng} \mathrm{mL}^{-1}$ individuals of respective $\mathrm{cMb}$ and CRP-spiked serum are comparable to the pure normal human serum response $\left(\Delta R_{\mathrm{et}}=95.9 \pm 17.2 \Omega\right.$ $\mathrm{cm}^{2}$ ), but they are far below the response $\left(\Delta R_{\mathrm{et}}=869.7 \pm 46.4 \Omega \mathrm{cm}^{2}\right)$ of bioelectrode to the same concentration of cTnI-spiked serum. This has further been confirmed with the negative control which also shows a comparable response $\left(\Delta R_{\mathrm{et}}=119 \pm 11.6 \Omega \mathrm{cm}^{2}\right)$ nearby to that of the pure human serum. The response of $\Delta R_{\mathrm{et}}=220 \pm 29.7 \Omega \mathrm{cm}^{2}$ to the lowest $1 \mathrm{pg} \mathrm{mL}^{-1} \mathrm{cTnI}$ concentration is about 2.3 times higher than the corresponding response to pure human serum. This lowest detection of $\mathrm{cTnI}$ is much smaller than the reported lowest detection level of $5.0 \mathrm{ng} \mathrm{mL}^{-1} \mathrm{cTnI}$ from the conventional ELISA method [31], which is signified the importance of the conducting polymer as a biomolecular linker over a nanocarbon hybrid for a better probe orientation. These results predicted a good specificity of the bioelectrode for cTnI. The reproducibility of the bioelectrode was evaluated in terms of relative standard deviation measured at individual $\mathrm{cTnI}$ concentration on three different bioelectrodes prepared in the same manner under identical conditions. It is shown as error bars in the calibration curve.

The measured RSD values of $\sim 2.8-13.4 \%$ at individual cTnI concentration were found to be within the acceptable error range under the tested conditions, suggesting a reproducible assay. The stability of the bioelectrode in solution was also investigated by taking repeated measurements (5-6 times) with a fixed concentration $\left(1.0 \mathrm{ng} \mathrm{mL}^{-1}\right.$ ) of cTnI sample. It was found to be within the reasonable range of consistent response, indicating to a good bioelectrode stability in solution.

\section{Conclusion}

We report a hybrid PPy-PPa/G-CNTs/GCE bioelectrode for ultrasensitive detection of cTnI. The pendant carboxyl groups of the polymer allowed efficient biomolecular 
immobilization of anti-cTnI on the electroactive G-CNTs, which is suitable for fabrication of a low-frequency impedimetric immunosensor. The modification of G-CNTs with conducting polymer makes prominent changes in the surface morphology and electrochemical characteristics with a capacitive behaviour without any diffusive feature. This biomolecular immobilization was modified to a nearly homogenous surface $(n=0.88)$ with a dominant charge transfer characteristic at the same frequency of $1.0 \mathrm{~Hz}$. It is useful for the quantitative analysis of analyte at low-frequency region. The synergistic combination of physical and electrochemical characteristics of both the conducting polymer and 2D-graphene with pillared G-CNTs hybrid results into the detection of cTnI concentration as low as $1.0 \mathrm{pg} \mathrm{mL}^{-1}$ (34.4 $\mathrm{fM}$ ) in spiked human serum, and the sensitivity is high of $167.8 \pm 14.2 \Omega \mathrm{cm}^{2}$ per decade of cTnI. Our results may be a reliable foundation of impedance immunosensor in detection of enzyme, protein, or DNA.

Acknowledgments The authors are grateful to the Director of the National Physical Laboratory, New Delhi, India for providing research facilities. Shobhita Singal is thankful to CSIR for providing a senior research fellowship. We are also thankful to Mr. V. K. Tanwar and Mr. Vikash Sharma for technical help.

\section{Compliance with Ethical Standards}

Conflict of interest No potential conflict of interest was reported by the authors.

Open Access This article is distributed under the terms of the Creative Commons Attribution 4.0 International License (http://crea tivecommons.org/licenses/by/4.0/), which permits unrestricted use, distribution, and reproduction in any medium, provided you give appropriate credit to the original author(s) and the source, provide a link to the Creative Commons license, and indicate if changes were made.

\section{References}

1. J. Gong, X. Miao, T. Zhou, L. Zhang, An enzymeless organophosphate pesticide sensor using Au-nanoparticle decorated graphene hybrid nanosheet as solid-phase extraction. Talanta 85(3), 1344-1349 (2011). doi:10.1016/j.talanta.2011.06. 016

2. J. Lu, I. Do, L.T. Drzal, R.M. Worden, I. Lee, Nanometal-decorated exfoliated graphite nanoplatelet based glucose biosensors with high sensitivity and fast response. ACS Nano 2(9), 1825-1832 (2008). doi:10.1021/nn800244k

3. X. Sun, L. Qiao, X. Wang, A novel immunosensor based on Au nanoparticles and polyaniline/multiwall carbon nanotubes/chitosan nanocomposite film functionalized interface. Nano-Micro Lett. 5(3), 191-201 (2013). doi:10.1007/BF03353750

4. D.H. Kim, J.A. Wiler, D.J. Anderson, D.R. Kipke, D.C. Martin, Conducting polymers on hydrogel-coated neural electrode provides sensitive neural recordings in auditory cortex. Acta Biomater. 6(1), 57-62 (2010). doi:10.1016/j.actbio.2009.07.034
5. S.M. Richardsons, J.L. Hendricks, B. Foster, Polymerization of the conducting polymer poly (3,4-ethylenedioxythiophene) (PEDOT) around living neural cells. Biomaterials 28(8), 1539-1552 (2007). doi:10.1016/j.biomaterials.2006.11.026

6. A. Ramanavicius, A. Ramanaviciene, A. Malinauskas, Electrochemical sensors based on conducting polymer-polypyrrole. Electrochim. Acta 51, 6025-6037 (2006). doi:10.1016/j.electacta. 2005.11.052

7. Y.P. Hsiao, Y.W. Su, J.R. Cheng, S.H. Cheng, Electrochemical determination of cysteine based on conducting polymers/gold nanoparticles hybrid nanocomposites. Electrochim. Acta 56(20), 6887-6895 (2011). doi:10.1016/j.electacta.2011.06.031

8. J.W. Lee, F. Serna, J. Nickels, C.E. Schimdt, Carboxylic acidfunctionalized conductive polypyrrole as a bioactive platform for cell adhesion. Biomacromolecules 7(6), 1692-1695 (2006). doi:10.1021/bm060220q

9. L.F.Q.P. Marchesi, F.R. Simoes, L.A. Pocrifka, E.C. Pereira, Investigation of polypyrrole degradation using electrochemical impedance spectroscopy. J. Phys. Chem. B 115(31), 9570-9575 (2011). doi:10.1021/jp2041263

10. W. Chen, Z. Lu, C.M. Li, Sensitive human interleukin 5 impedimetricimmunosensor based on polypyrrole-pyrrolepropylic acid-gold nanocomposite. Anal. Chem. 80(22), 8485-8492 (2008). doi:10.1021/ac8012225

11. D. Wang, W. Hu, Y. Xiong, Y. Xu, C.M. Li, Multifunctionalized reduced graphene oxide-doped polypyrrole/pyrrolepropylic acid nanocomposite impedimetricimmunosensor to ultra-sensitively detect small molecular aflatoxin B1. Biosens. Bioelectron. 63, 185-189 (2015). doi:10.1016/j.bios.2014.06.070

12. K.S. Novoselov, A.K. Geim, S.V. Morozov, D. Jiang, Y. Zhang, S.V. Dubonos, I.V. Grigorieva, A.A. Firsov, Electric field effect in atomically thin carbon films. Science 306(5696), 666-669 (2004). doi:10.1126/science.1102896

13. A. Bonnani, A.H. Loo, M. Pumera, Graphene for impedimetric biosensing. Anal. Chem. 37(37), 12-21 (2012). doi:10.1016/j. trac.2012.02.011

14. Y.M. Lin, C. Dimitrakopoulos, K.A. Jenkins, D.B. Farmer, H.Y. Chiu, A. Grill et al., 100-GHz transistors from wafer-scale epitaxial graphene. Science 327(5966), 662 (2010). doi:10.1126/sci ence. 1184289

15. N. Zhang, M.Q. Yang, S. Liu, Y. Sun, Y.J. Xu, Waltzing with the versatile platform of graphene to synthesize composite photocatalysts. Chem. Rev. 115(18), 10307-10377 (2015). doi:10. 1021/acs.chemrev.5b00267

16. N. Zhang, Y.J. Xu, The endeavour to advance graphene-semiconductor composite-based photocatalysis. Cryst. Eng. Commun. 18(1), 24-37 (2016). doi:10.1039/C5CE01712B

17. N. Zhang, Y. Zhang, Y.J. Xu, Recent progress on graphene-based photocatalysts: current status and future perspectives. Nanoscale 4(19), 5792-5813 (2012). doi:10.1039/c2nr31480k

18. S. Paulson, A. Helser, M.B. Nardelli, R.M. Taylor, M. Falvo, R. Superfine, S. Washburn, Tunable resistance of a carbon nanotubegraphite interface. Science 290(5497), 1742-1744 (2000). doi:10. 1126/science.290.5497.1742

19. F.D. Novaes, R. Rurali, P. Ordejon, Electronic transport between graphene layers covalently connected by carbon nanotubes. ACS Nano 4(12), 7596-7602 (2010). doi:10.1021/nn102206n

20. Y.S. Kim, K. Kumar, F.T. Fisher, E. Yang, Out-of-plane growth of CNTs on graphene for supercapacitor applications. Nanotechnology 23(1), 015301 (2012). doi:10.1088/0957-4484/23/1/ 015301

21. K.Y. Hwa, B. Subramani, Synthesis of zinc oxide nanoparticles on graphene-carbon nanotube hybrid for glucose biosensor applications. Biosens. Bioelectron. 62(22), 127-133 (2014). doi:10.1016/j.bios.2014.06.023 
22. B. Kaur, T. Pandiyan, B. Satpati, R. Srivastava, Simultaneous and sensitive determination of ascorbic acid, dopamine, uric acid, and tryptophan with silver nanoparticles-decorated reduced graphene oxide modified electrode. Colloid Surf. B 111(6), 97-106 (2013). doi:10.1016/j.colsurfb.2013.05.023

23. P. Ammann, M. Pfisterer, T. Fehr, H. Rickli, Raised cardiac troponins; causes extend beyond acute coronary syndromes. Br. Med. J. 328(7447), 1028-1029 (2004). doi:10.1136/bmj.328. 7447.1028

24. S. Ko, B. Kim, S.S. Jo, S.Y. Oh, J.K. Park, Electrochemical detection of cardiac troponin I using a microchip with the surface-functionalized poly (dimethylsiloxane) channel. Biosens. Bioelectron. 23(1), 51-59 (2007). doi:10.1016/j.bios.2007.03.013

25. A. Qureshi, Y. Gurbuz, J.H. Niazi, Biosensors for cardiac biomarkers detection: a review. Sens. Actuat. B 171-172, 62-76 (2012). doi:10.1016/j.snb.2012.05.077

26. R.K. Rajesh, A. Paul, Mulchandani, Platinum nanoflowers decorated three-dimensional graphene-carbon nanotubes hybrid with enhanced electrocatalytic activity. J. Power Sources 223(1), 23-29 (2013). doi:10.1016/j.jpowsour.2012.08.088

27. Y. Li, P. Wang, L. Wang, X. Lin, Over oxidized polypyrrole film directed single-walled carbon nanotubes immobilization on glassy carbon electrode and its sensing applications. Biosens. Bioelectron. 22(12), 3120-3125 (2007). doi:10.1016/j.bios.2007. 02.001

28. B. Rajib, M. Larif, G. Mouhssine, A. Elmidaoui, M.E. Touhami, A. Chaouch, Valorization of polyphenols extracted from olive mill wastewater as ecological corrosion inhibitor on carbon steel in acid medium. Der Pharm. Chem. 8(2), 145-153 (2016)

29. B. Derkus, M. Ozkan, K.C. Emregul, E. Emregul, Single frequency analysis for clinical immunosensor design. RSC Adv. 6(1), 281-289 (2016). doi:10.1039/C5RA23783A
30. M. Bart, E.C.A. Stigter, H.R. Stapert, G.J. Jong, W.P. Bennekom, On the response of a labl-free interferon $-\gamma$ immunosensor utilizing electrochemical impedance spectroscopy. Biosens. Bioelectron. 21(1), 49-59 (2005). doi:10.1016/j.bios.2004.10.009

31. A. Periyakaruppan, R.P. Gandhiraman, M. Meyyappan, J.E. Koehne, Label-free detection of cardiac troponin-I using carbon nanofiber based nanoelectrode array. Anal. Chem. 85(8), 3858-3863 (2013). doi:10.1021/ac302801z

32. Z.R. Guo, C.R. Gu, X. Fan, Z.P. Bian, H.F. Wu, D. Yang, N. Gu, J.N. Zhang, Fabrication of anti-human cardiac troponin I immunogold nanorods for sensing acute myocardial damage. Nanoscale Res. Lett. 4(12), 1428-1433 (2009). doi:10.1007/ s11671-009-9415-6

33. A.J.S. Ahammad, Y.H. Choi, K. Koh, J.H. Kim, J.J. Lee, M. Lee, Electrochemical detection of cardiac biomarker troponin I at gold nanoparticle-modified ITO electrode by using open circuit potential. Int. J. Electrochim. Sci. 6(6), 1906-1916 (2011)

34. A.A. Shumkov, E.V. Suprun, S.Z. Shatinina, A.V. Lisitsa, V.V. Shumyantseva, A.I. Archakov, Gold and silver nanoparticles for electrochemical detection of cardiac troponin I based on stripping voltammetry. J. Bionanosci. 3(2), 216-222 (2013). doi:10.1007/ s12668-013-0090-9

35. W.Y. Wu, Z.P. Bian, W. Wang, W. Wang, J.J. Zhu, PDMS gold nanoparticle composite film-based silver enhanced colorimetric detection of cardiac troponin I. Sens. Actuat. B 147(1), 298-303 (2010). doi:10.1016/j.snb.2010.03.027

36. Y. Ryu, Z. Jin, M.S. Kang, H.S. Kim, Increase in the detection sensitivity of a lateral flow assay for a cardiac marker by oriented immobilization of antibody. Biochip J. 5, 193-198 (2011). doi:10.1007/s13206-011-5301-2 